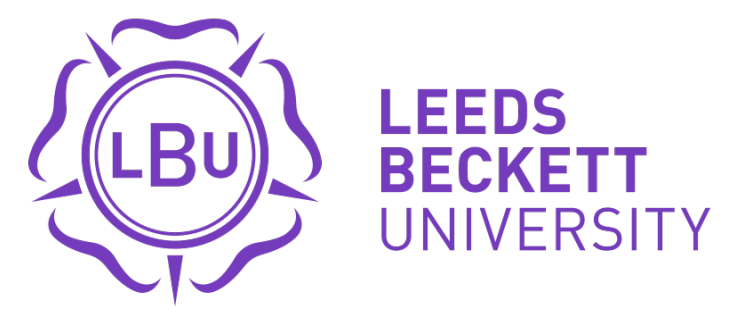

Citation:

Chung, CP and Beggs, CB and Wang, PN and Bergsland, N and Shepherd, S and Cheng, CY and Ramasamy, DP and Dwyer, MG and Hu, HH and Zivadinov, R (2014) Jugular venous reflux and white matter abnormalities in Alzheimer's disease: a pilot study. Journal of Alzheimer's disease : JAD, 39 (3). 601 - 609. ISSN 1387-2877 DOI: https://doi.org/10.3233/jad-131112

Link to Leeds Beckett Repository record:

https://eprints.leedsbeckett.ac.uk/id/eprint/2376/

Document Version:

Article (Accepted Version)

The aim of the Leeds Beckett Repository is to provide open access to our research, as required by funder policies and permitted by publishers and copyright law.

The Leeds Beckett repository holds a wide range of publications, each of which has been checked for copyright and the relevant embargo period has been applied by the Research Services team.

We operate on a standard take-down policy. If you are the author or publisher of an output and you would like it removed from the repository, please contact us and we will investigate on a case-by-case basis.

Each thesis in the repository has been cleared where necessary by the author for third party copyright. If you would like a thesis to be removed from the repository or believe there is an issue with copyright, please contact us on openaccess@leedsbeckett.ac.uk and we will investigate on a case-by-case basis. 


\section{Jugular venous reflux and white matter abnormalities in Alzheimer's \\ disease: A pilot study}

Chih-Ping Chung ${ }^{\mathrm{a}, \mathrm{b}}$, Clive Beggs ${ }^{\mathrm{c}}$, Pei-Ning Wang, ${ }^{\mathrm{a}, \mathrm{b}}$ Niels Bergsland $^{\mathrm{d}}$, Simon Shepherd ${ }^{\mathrm{c}}$,

Chun-Yu Cheng ${ }^{\mathrm{a}, \mathrm{b}, \mathrm{e}}$, Deepa P. Ramasamy ${ }^{\mathrm{d}}$, Michael G. Dwyer ${ }^{\mathrm{d}}$, Han-Hwa Hu${ }^{\mathrm{a}, \mathrm{b}}$, and Robert Zivadinov $^{\mathrm{d}^{*}}$

${ }^{a}$ Department of Neurology, Taipei Veterans General Hospital, Taipei, Taiwan

${ }^{b}$ Department of Neurology, National Yang Ming University of Medicine, Taipei, Taiwan

${ }^{c}$ Centre for Infection Control and Biophysics, University of Bradford, Bradford, UK

${ }^{d}$ Buffalo Neuroimaging Analysis Center, Department of Neurology, University at Buffalo, Buffalo, NY, USA

${ }^{e}$ Institute of Brain Science, National Yang-Ming University School of Medicine, Taipei, Taiwan.

Dr. Wang and Dr. Zivadinov have equal authorship contributions

Running title: Jugular venous reflux in Alzheimer's disease

Correspondence to Dr. Zivadinov, Professor of Neurology, School of Medicine and Biomedical Sciences, State University of New York, Director, Buffalo Neuroimaging Analysis Center, 100 High St., Buffalo, NY 14203, USA.

E-mail: rzivadinov@bnac.net

All authors have disclosed no conflict of interest to the manuscript.

Abstract count:252, Word count: 2517, Number of Tables: 3, Number of supplementary appendices: 2, Number of references: 43. 


\section{ABSTRACT}

To determine whether jugular venous reflux (JVR) is associated with cerebral white matter changes (WMCs) in individuals with Alzheimer's disease (AD), we studied 12 AD patients 24 mild cognitive impairment (MCI) patients and 17 elderly controls age- and sex-matched. Duplex ultrasonography and 1.5T MRI scanning was applied to quantify cerebral WMCs [T2 white matter (WM) lesion and dirty-appearing-white-matter (DAWM)]. Subjects with severe JVR had more frequently hypertension ( $p=0.044)$, more severe WMC, including increased total $(p=0.047)$ and periventricular DAWM volumes $(p=0.008)$ and a trend for increased CSF volumes $(\mathrm{p}=0.067)$ compared with the other groups. A significantly decreased $(65.8 \%)$ periventricular DAWM volume $(p=0.01)$ in the JVR positive AD individuals compared with their JVR negative counterparts was detected. There was a trend for an increased periventricular and subcortical T2 WMC lesion volumes in the JVR positive AD individuals compared with their JVR negative counterparts $(p=0.073)$. This phenomenon was not observed in either the control or MCI groups. In multiple regression analysis, the increased periventricular WMC lesion volume and decreased DAWM volume resulted in $85.7 \%$ sensitivity and $80 \%$ specificity for distinguishing between JVR positive and negative AD patients. These JVR-WMC association patterns were not seen in the control and MCI groups. Therefore, this pilot study suggests that there may be an association between JVR and WMCs in $\mathrm{AD}$ patients, implying cerebral venous outflow impairment, might play a role in the dynamics of WMCs formation in $\mathrm{AD}$ patients, particularly in the periventricular regions. Further longitudinal studies are needed to confirm and validate our findings.

Keywords: Alzheimer's disease, leukoaraiosis, jugular veins, magnetic resonance imaging, Doppler ultrasonography 
Glossary: $\mathrm{WM}=$ white matter; $\mathrm{WMC}=$ white matter change; $\mathrm{ARWMC}=$ age-related white matter change; $\mathrm{AD}=$ Alzheimer's disease $\mathrm{MCI}=$ mild cognitive impairment $\mathrm{JVR}=$ jugular venous reflux; DAWM = dirty-appearing white matter; $\mathrm{LV}=$ lateral ventricle; $\mathrm{CSF}=$ cerebrospinal fluid. 


\section{INTRODUCTION}

White matter changes (WMCs) with punctate and/or confluent WM lesions in the periventricular and/or deep subcortical regions, revealed as hyperintensities on T2-weighted or fluid-attenuated inversion recovery (FLAIR) MRI are strongly related to aging [1]. These age-related WMCs (ARWMCs) are associated with occurrences of different types of dementia including Alzheimer's disease (AD) and predict faster cognitive decline not only in the general population, but also in $\mathrm{AD}$ patients $[2,3]$.

Jugular venous reflux (JVR), characterized by a retrograde flow in internal jugular veins (IJVs) during Valsalva maneuvers (VM) or spontaneously at rest, is found more frequently in the elderly [4-6]. Recently, it has been found that JVR is associated with ARWMC, particularly in posterior brain regions [7]. Retrograde-transmitted venous pressure associated with JVR in the elderly is thought to result in accumulated insults to the cerebral vasculature, small vessel structure/function, and/or the brain-blood barrier (BBB), leading to WM damage [7, 8]. Compared with non-demented individuals, WMCs are more prevalent and severe in AD patients [9-14]. In this study, we tested the hypothesis that JVR might be involved in the dynamics of WMC formation in AD by evaluating the relationship between the severity of JVR and WMC in patients with AD.

\section{MATERIAL AND METHODS}

\section{Subjects}

Between December 2008 and April 2010, Taiwanese residents consecutively admitted to a memory clinic at Taipei Veterans General Hospital, Taiwan due to subjective memory complaints were assessed for inclusion. Neurologists performed clinical and neurologic evaluations in all participants. A standard set of neuropsychological assessments including the Mini-Mental State Examination (MMSE) and Clinical Dementia Rating (CDR) scales were used. 
Subjects eligible for participation in the current study were 55 years of age or older, had a CDR score $\leq 1$ (as an assurance that they could cooperate during the VM for JVR detection), and were willing to receive brain MRI and neck duplex ultrasonography. Exclusion criteria for all subjects were a past history of stroke, ischemic heart disease, congestive heart disease, valvular heart disease, cardiac arrhythmia, pulmonary diseases, or malignancy, and having brain MRI of insufficient quality for performing quantitative volumetric analysis.

Vascular risk factors were defined according to international guidelines and prospectively identified using all available information including medical charts, laboratory results, patient interviews, and neurological examinations (Supplementary Appendix 1) [15].

Subjects were classified into AD, mild cognitive impairment (MCI) or control groups according to the criteria of National Institute of Neurological and Communicative Disorders and Stroke/Alzheimer's Disease and Related Disorders Association and by Petersen et al.'s study revised by the Stockholm consensus group [16-18]. The hospital's Institutional Review Boards approved the study and each included participant or his/her caregiver provided informed consent.

\section{Color-coded duplex ultrasonography: JVR determination}

Ultrasonographic determination of JVR has been described previously (Supplementary Appendix 2) [6, 7]. JVR could be detected spontaneously at rest or only during VM. We graded the severity of JVR into three groups: subjects with no JVR on both internal jugular vein (IJV) sides were classified as a no-JVR group. Subjects with JVR detected on either or both IJV sides during VM only, or subjects with spontaneous JVR on one IJV side and no JVR on the other IJV side were graded as the mild-JVR group. Subjects with JVR detected on one IJV side spontaneously at rest in combination with JVR detected during VM or spontaneously at rest in the other side were graded as the severe-JVR group [7]. 


\section{MRI acquisition}

All study participants were scanned on 1.5T MRI (Excite II; GE Medical Systems,

Milwaukee, WI). MRI brain sequences included axial two dimensional (2D) T1- and T2-

weighted imaging (WI), FLAIR and three dimensional (3D) T1 spoiled gradient echo (SPGR)

pulse sequences. The images were acquired with the following sequence parameters: for 3D

T1 the repetition time (TR) was $8.5 \mathrm{~ms}$, echo time (TE) $1.8 \mathrm{~ms}$, inversion time (TI) $400 \mathrm{~ms}$, flip angle (FA) $15^{\circ}$, slice thickness $1.5 \mathrm{~mm}$, field of view (FOV) $260 \mathrm{~mm}$ x $260 \mathrm{~mm}$ and matrix 256 x 256; for T2 the TR was 3700 ms, TE 102 ms, echo train length 19, slice thickness 3mm, FOV 240mm x 240mm and matrix 256 x 256; for FLAIR: TR 9000 ms, TE 120 ms, TI 2250 ms, slice thickness 3mm, FOV 240mm x 240mm and matrix 256x256.

\section{MRI image analysis}

The MRI analysis personnel were blinded to the subjects' demographic and clinical characteristics.

We classified WMCs as belonging to two groups of signal abnormalities based on FLAIR/T2-WI signal intensity: T2 white matter (WM) lesion and dirty-appearing-whitematter (DAWM). The T2 WM lesion number and volume on T2-WI were outlined using a semi-automated edge detection contouring/thresholding technique as described previously [19]. Regional localization of WM lesion volume was determined in the periventricular and deep subcortical regions. We also outlined areas of DAWM and calculated their number and volume. The DAWM was defined as a, non-focal area of signal increase on the FLAIR/T2WI, with a subtly increased signal intensity compared with the signal intensity of normal appearing WM, as previously proposed [20]. The DAWM showed relatively diffuse border compared with focal T2 WM lesions and was tapered off toward the normal appearing WM (Fig. 1). The regional localization of DAWM volume was also determined in the 
periventricular and deep subcortical regions. The data are presented separately for number and volume of total and regional T2 WM lesions as well as DAWM.

In addition, we used Structural Image Evaluation, using Normalisation, of Atrophy, cross-sectional (SIENAX, version 2.6) to estimate normalized lateral ventricular volume (NLVV), and normalized CSF volume, as previously described [21].

\section{Statistical analysis}

Statistical analysis was undertaken using a combination of Statistical Package for the Social Sciences (SPSS, IBM, Armonk, New York, USA) and in-house algorithms written in Matlab (Mathworks, Natick, Mass) with the aim of evaluating the impact of JVR on the respective MRI variables.

Parametric and non-parametric analyses were performed on the respective study cohorts to identify significant differences between the various study groups, including disease groups, JVR severity score and JVR presence. In order to identify MRI variables that were associated with the presence of JVR we obtained plots of the logit vs. logistic function of the multiple regression analysis in the respective study groups.

Given the exploratory pilot nature of the study, values of $p<0.05$ were considered statistically significant and $p<0.1$ was considered a trend.

\section{RESULTS}

Eighty-four subjects [57-93 years of age; mean(SD): 79.77(7.41) years; 33 women; 31 control subjects, 33 MCI subjects, and 20 AD subjects] were eligibly enrolled according to our criteria. To match age and gender in each disease group, fifty three subjects [64-91 years of age; mean(SD): 81.5 (4.50) years; 22 women] of them were enrolled for further analysis. There were 17 control subjects, $24 \mathrm{MCI}$ subjects, and 12 AD subjects (Table 1). All the AD patients met the diagnostic criteria for $\mathrm{AD}$ and $\mathrm{CDR}=1$; and all $\mathrm{MCI}$ patients met the diagnostic criteria for MCI and had a $\mathrm{CDR}=0.5$. Twenty two subjects $(41.5 \%)$ had right-sided 
JVR (all detected during VM) and 23 subjects (43.4\%) had left-sided JVR (9 detected spontaneously at rest and 14 detected during VM, Table 2). There were 12 subjects (22.6\%) with bilateral JVR; among them, 5 had unilateral spontaneous JVR at rest with contralateral VM-induced JVR and 7 had bilateral VM-induced JVR. Twenty subjects (37.7\%) presented with JVR on neither side.

Table 1 shows the comparisons of clinical characteristics and MRI variables between control, MCI and AD groups. From this it can be seen that for all but three study variables there was no significant difference between the respective groups. The only exceptions to this were: the MMSE score, which was significantly lower in the AD group $(p<0.001)$; the number of years in education, which was on average approximately 3 years less in the MCI and AD groups ( $p=0.033$ ); and hyperlipidemia, which had a higher incidence in the AD group $(p=0.014)$.

The clinical characteristics and MRI variables between the three JVR-graded groups are presented in Table 2, which also shows the frequencies of vascular risk factors. The three JVR-graded groups were closely age-matched and had similar clinical characteristics, with the exception of the incidence of hypertension, which was significantly greater in the mild and severe JVR groups $(p=0.044)$. There was a general trend for an increased WMC number and volume in the severe-JVR group that reached significance for the total DAWM $(p=0.047)$ and periventricular DAWM volume ( $p=0.008)$. The more severe JVR group also exhibited a trend for increased CSF volumes compared with the other groups $(\mathrm{p}=0.067)$.

Further investigation, using a 2-tailed Mann Whitney U-test (Table 3), revealed a significantly decreased $(65.8 \%)$ periventricular DAWM volume $(p=0.01)$ in the JVR positive AD individuals compared with their JVR negative counterparts. There was also a trend for an increased periventricular and subcortical T2 WMC lesion volume in the JVR positive AD 
individuals compared with their JVR negative counterparts $(p=0.073)$. This phenomenon was not observed in the either the control or MCI groups.

Figure 2 shows a plot of the logit vs. logistic function of the multiple regression analysis for the $\mathrm{AD}$ patients group using the periventricular $\mathrm{T} 2 \mathrm{WMC}$ volume $(\mathrm{B}=0.0004)$ and periventricular DAWM volume $(B=-0.0039)$ vs. the JVR positivity score. This resulted in sensitivity of $85.7 \%$ and specificity of $80 \%$ for distinguishing between JVR positive and negative AD patients. Similar analysis revealed that none of the MRI variables were significantly associated with JVR positivity score in the control and MCI groups.

\section{DISCUSSION}

The present pilot study was of limited scope, having a small sample size with only few subjects who presented with severe JVR. Therefore, our findings should be interpreted with caution and no firm conclusions should be made regarding the general applicability of our findings at this moment in time. Future studies including larger sample size and more MCI and $\mathrm{AD}$ patients are needed to confirm the validity of our findings.

The principal finding of the present study is that JVR appears to be associated with changes in the DAWM, particularly in the periventricular region. Increased DAWM volume, particularly in the periventricular region was associated with a more severe JVR score. DAWM is defined as a region of intermediate signal intensity between that of T2 WM lesions and that of normal-appearing white-matter [20, 23-27]. The measurement of DAWM is relatively unexplored territory in neurodegenerative disorders and aging. To the best of our knowledge, the present study is the first of its kind to explore measurement of the DAWM in elderly. Most of the work on DAWM has been performed in patients with multiple sclerosis [20, 23-27], and although the concept of DAWM remains largely under investigation, it appears to have potential as a diagnostic metric. Image-pathology correlation studies reveal DAWM, compared with T2 WM lesions, to be associated with mild blood brain barrier 
(BBB) breakdown and myelin loss [20, 23-27]. Therefore, it can be suggested that DAWM may represent an earlier stage of WM pathology in elderly $[25,26]$, which is followed at a later stage, by T2 WM lesion formation [35-38]. Indeed, using WMC lesion volumes and DAWM it was possible to distinguish between the JVR positive and negative AD patients, with high degree of accuracy (sensitivity $=85.7 \%$ and specificity $=80 \%$ ) and minimal overlap (Figure 2). These findings suggests that in the AD group, DAWM areas may have converted into T2 WM lesions at a greater rate in subjects with more severe JVR (Table 3). However, future longitudinal studies are necessary to elucidate the chronological JVR effects on WMC formation in AD patients.

The present study suggests that periventricular WMCs may be associated with JVR (Tables 2 and 3). This regional-association was not shown in our previous study [7], possibly because: (1) the volumetric and DAWM approaches used in this study were more sensitive to small group differences than the WMC visual-rating scale used previously [20, 22-27]; or (2) there were differences in the severe-JVR populations of the groups that were used in the two studies [7]. Why the periventricular region in AD patients could be influenced by JVR is something unknown at this time. However, there is evidence that venous drainage anomalies can influence CSF dynamics, slowing down CSF absorption into the superior sagittal sinus [39]. The lateral ventricles represent the major reservoir of CSF in the brain. It has been proposed that retrograde-transmitted venous pressure, caused by JVR, may induce venous hypertension in the superior sagittal sinus, resulting in decreased CSF absorption and larger lateral ventricle volume [28-31] and CSF volume, as evidenced in the present study. However, this association has to be further investigated.

The etiology of WMC in AD is still uncertain. Our pilot results suggest that DAWM may be a precursor to $\mathrm{T} 2$ lesion formation and that transition between the two might be accelerated by the presence of JVR. It may be that JVR alters the intracranial biomechanics 
interacting synergistically with other AD pathologies. That these associations were not observed in either the MCI subjects or controls may suggest that JVR may play some role in late $\mathrm{AD}$ pathophysiology. It has been reported that retrogradely transmitted venous hypertension in AD patients associated with JVR might: (1) decrease cerebral perfusion, damage small vessel structure/function, and possibly damage the BBB [8]; or (2) impair CSF absorption, thus reducing CSF net flow, enlarging ventricles, and possibly lowering $\beta$ amyloid clearance with the result that it accumulates in the periventricular tissues [32-34]. Either one or both of these mechanisms might contribute to WMC formation in AD patients. However, at this stage it is not possible to ascertain this, and further investigation is required before any causal link between JVR in AD pathology can be claimed. Our findings may serve as an important pilot data in this direction.

It is noticeable that JVR appears to be associated with certain cardiovascular risk factors, e.g. hypertension. Dolic et al. [40] studied 240 healthy individuals and found that the presence of heart disease, especially heart murmurs, obesity and cigarette smoking were associated with an increased prevalence of extracranial venous abnormalities. These results, in addition to the findings from the present study, may imply that cardiovascular or systemic metabolic disorders may be involved in the pathophysiology of venous (especially IJV) hemodynamic abnormalities. Besides, it is known that patients with hypertension have less venous distensibility $[41,42]$. One characteristic specific for venous walls is that they have greater distensibility than arterial walls which makes them able to accommodate a greater range of increased venous pressure [43]. Decreased venous distensibility in hypertension might reduce this pressure buffer ability in IJV and repetitive higher venous pressure might damage IJV valvular structure and its competence, leading to more frequent JVR seen in patients with hypertension. 
It should be noted that this pilot study had a relatively small sample size and that future larger case-control studies are needed to elucidate the differences of WMC severity between $\mathrm{AD}, \mathrm{MCI}$ and control groups. In addition, another limitation of the study is related to its cross-sectional nature, and further longitudinal studies are needed to determine temporal relationship between JVR and WMC lesion formation in relation to DAWM, particularly in the periventricular regions.

In conclusion, the findings of our pilot study showed that cerebral venous drainage impairment may play some role in the pathophysiology of WMC in the AD patients.. 


\section{REFERENCES}

[1] Hachinski VC, Potter P, Merskey H (1987) Leuko-araiosis. Arch Neurol 44, 21-23.

[2] Debette S, Markus HS (2010) The clinical importance of white matter hyperintensities on brain magnetic resonance imaging: systematic review and meta-analysis. $B M J$ 341, c3666.

[3] Brickman AM, Muraskin J, Zimmerman ME (2009) Structural neuroimaging in Altheimer's disease: do white matter hyperintensities matter? Dialogues Clin Neurosci 11, 181-190.

[4] Tanaka T, Uemura K, Takahashi M, Takehara S, Fukaya T, Tokuyama T, Satoh A, Ryu H (1993) Compression of the left brachiocephalic vein: cause of high signal intensity of the left sigmoid sinus and internal jugular vein on MR images. Radiology 188, 355-361.

[5] Akkawi NM, Agosti C, Borroni B, Rozzini L, Magoni M, Vignolo LA, Padovani A (2002) Jugular valve incompetence: a study using air contrast ultrasonography on a general population. J Ultrasound Med 21, 747-751.

[6] Chung CP, Lin YJ, Chao AC, Lin SJ, Chen YY, Wang YJ, Hu HH (2010) Jugular venous hemodynamic changes with aging. Ultrasound Med Biol 36, 1776-1782.

[7] Chung CP, Wang PN, Wu YH, Tsao YC, Sheng WY, Lin KN, Lin SJ, Hu HH (2011) More severe white matter changes in the elderly with jugular venous reflux. Ann Neurol 69, 553-559.

[8] Chung CP, Hu HH (2010) Pathogenesis of leukoaraiosis: role of jugular venous reflux. Med Hypotheses 75, 85-90.

[9] Scheltens P, Barkhof F, Valk J, Algra PR, van der Hoop RG, Nauta J, Wolters EC (1992) White matter lesions on magnetic resonance imaging in clinically diagnosed Alzheimer's disease. Evidence for heterogeneity. Brain 115, 735-748.

[10] Kalaria RN (2000) The role of cerebral ischemia in Alzheimer's disease. Neurobiol Aging 21, 321-330. 
[11] Meyer JS, Kawamura J, Terayama Y (1992) White matter lesions in the elderly. J Neur Sci 110, 1-7.

[12] Rezek DL, Morris JC, Fulling KH, Gado MH (1987) Periventricular white matter lucencies in senile dementia of the Alzheimer type and in normal aging. Neurology 37, 13651368.

[13] Yoshita M, Fletcher E, Harvey D, Ortega M, Martinez O, Mungas DM, Reed BR, DeCarli CS (2006) Extent and distribution of white matter hyperintensities in normal aging, MCI, and AD. Neurology 67, 2192-2198.

[14] Pettersen JA, Sathiyamoorthy G, Gao FQ, Szilagyi G, Nadkarni NK, St George-Hyslop P, Rogaeva E, Black SE (2008) Microbleed topography, leukoaraiosis, and cognition in probable Alzheimer disease from the Sunnybrook dementia study. Arch Neurol 65, 790-795. [15] Goldstein LB, Adams R, Alberts MJ, Appel LJ, Brass LM, Bushnell CD, Culebras A, Degraba TJ, Gorelick PB, Guyton JR, Hart RG, Howard G, Kelly-Hayes M, Nixon JV, Sacco RL; American Heart Association/American Stroke Association Stroke Council; Atherosclerotic Peripheral Vascular Disease Interdisciplinary Working Group; Cardiovascular Nursing Council; Clinical Cardiology Council; Nutrition, Physical Activity, and Metabolism Council; Quality of Care and Outcomes Research Interdisciplinary Working Group; American Academy of Neurology (2006) Primary prevention of ischemic stroke: a guideline from the American Heart Association/American Stroke Association Stroke Council: cosponsored by the Atherosclerotic Peripheral Vascular Disease Interdisciplinary Working Group; Cardiovascular Nursing Council; Clinical Cardiology Council; Nutrition, Physical Activity, and Metabolism Council; and the Quality of Care and Outcomes Research Interdisciplinary Working Group: the American Academy of Neurology affirms the value of this guideline. Stroke 37, 1583-1633.

[16] McKhann G, Drachman D, Folstein M, Katzman R, Price D, Stadlan EM (1984) Clinical 
diagnosis of Alzheimer's disease: report of the NINCDS-ADRDA Work Group under the auspices of Department of Health and Human Services Task Force on Alzheimer's Disease. Neurology 34, 939-944.

[17] Petersen RC, Doody R, Kurz A, Mohs RC, Morris JC, Rabins PV, Ritchie K, Rossor M, Thal L, Winblad B (2001) Current concepts in mild cognitive impairment. Arch Neurol 58, 1985-1992.

[18] Artero S, Petersen R, Touchon J, Ritchie K (2006) Revised criteria for mild cognitive impairment: validation within a longitudinal population study. Dement Geriatr Cogn Disord 22, 465-470.

[19] Zivadinov R, Rudick RA, De Masi R, Nasuelli D, Ukmar M, Pozzi-Mucelli RS, Grop A, Cazzato G, Zorzon M (2001) Effects of IV methylprednisolone on brain atrophy in relapsingremitting MS. Neurology 57, 1239-1247.

[20] Seewann A, Vrenken H, van der Valk P, Blezer EL, Knol DL, Castelijns JA, Polman CH, Pouwels PJ, Barkhof F, Geurts JJ (2009) Diffusely abnormal white matter in chronic multiple sclerosis: imaging and histopathologic analysis. Arch Neurol 66, 601-609.

[21] Zivadinov R, Heininen-Brown M, Schirda CV, Poloni GU, Bergsland N, Magnano CR, Durfee J, Kennedy C, Carl E, Hagemeier J, Benedict RH, Weinstock-Guttman B, Dwyer MG (2012) Abnormal subcortical deep-gray matter susceptibility-weighted imaging filtered phase measurements in patients with multiple sclerosis: a case-control study. Neuroimage 59, 331339.

[22] van Straaten EC, Fazekas F, Rostrup E, Scheltens P, Schmidt R, Pantoni L, Inzitari D, Waldemar G, Erkinjuntti T, Mäntylä R, Wahlund LO, Barkhof F; LADIS Group (2006) Impact of white matter hyperintensities scoring method on correlations with clinical data: the LADIS study. Stroke 37, 836-840.

[23] Ge Y, Grossman RI, Babb JS, He J, Mannon LJ (2003) Dirty-appearing white matter in 
multiple sclerosis: volumetric MR imaging and magnetization transfer ratio histogram analysis. AJNR Am J Neuroradiol 24, 1935-1940.

[24] Chen SC, Chung HW, Liou M (2003) Measurement of volumetric lesion load in multiple sclerosis: moving from normal- to dirty-appearing white matter. AJNR Am J Neuroradiol 24, 1929-1930.

[25] Moore GR, Laule C, Mackay A, Leung E, Li DK, Zhao G, Traboulsee AL, Paty DW (2008) Dirty-appearing white matter in multiple sclerosis: preliminary observations of myelin phospholipid and axonal loss. J Neurol 255, 1802-1811.

[26] Laule C, Vavasour IM, Leung E, Li DK, Kozlowski P, Traboulsee AL, Oger J, Mackay AL, Moore GR (2011) Pathological basis of diffusely abnormal white matter: insights from magnetic resonance imaging and histology. Mult Scler 17, 144-150.

[27] Laule C, Pavlova V, Leung E, Zhao G, MacKay AL, Kozlowski P, Traboulsee AL, Li DK, Moore GR (2013) Diffusely abnormal white matter in multiple sclerosis: further histologic studies provide evidence for a primary lipid abnormality with neurodegeneration. $J$ Neuropathol Exp Neurol 72, 42-52.

[28] Schaller B, Graf R (2004) Cerebral venous infarction: the pathophysiological concept. Cerebrovasc Dis 18, 179-188.

[29] Zamboni P, Menegatti E, Weinstock-Guttman B, Schirda C, Cox JL, Malagoni AM, Hojanacki D, Kennedy C, Carl E, Dwyer MG, Bergsland N, Galeotti R, Hussein S, Bartolomei I, Salvi F, Zivadinov R (2009) The severity of chronic cerebrospinal venous insufficiency in patients with multiple sclerosis is related to altered cerebrospinal fluid dynamics. Funct Neurol 24, 133-138.

[30] Wu IH, Sheng WY, Hu HH, Chung CP. Jugular venous reflux could influence cerebral blood flow: a transcranial Doppler study (2011) Acta Neurol Taiwan 20, 15-21. [31] Chung CP, Hsu HY, Chao AC, Cheng CY, Lin SJ, Hu HH (2009) Jugular venous reflux 
affects ocular venous system in transient monocular blindness. Cerebrovasc Dis 29,122-129. [32] Ott BR, Cohen RA, Gongvatana A, Okonkwo OC, Johanson CE, Stopa EG, Donahue JE, Silverberg GD, Alzheimer's Disease Neuroimaging Initiative (2010) Brain ventricular volume and cerebrospinal fluid biomarkers of Alzheimer's disease. J Alzheimers Dis 20, 647-657. [33] Mawuenyega KG, Sigurdson W, Ovod V, Munsell L, Kasten T, Morris JC, Yarasheski KE, Bateman RJ (2010) Decreased clearance of CNS beta-amyloid in Alzheimer's disease. Science 330, 1774.

[34] Reed-Cossairt A, Zhu X, Lee HG, Reed C, Perry G, Petersen RB (2012) Alzheimer's disease and vascular deficiency: lessons from imaging studies and down syndrome. Curr Gerontol Geriatr Res 2012, 929734.

[35] Fazekas F, Chawluk JB, Alavi A, Hurtig HI, Zimmerman RA (1987) MR signal abnormalities at $1.5 \mathrm{~T}$ in Alzheimer's dementia and normal aging. AJR Am J Roentgenol 149:351-356.

[36] De Groot CJ, Bergers E, Kamphorst W, Ravid R, Polman CH, Barkhof F, van der Valk P (2001) Post-mortem MRI-guided sampling of multiple sclerosis brain lesions: increased yield of active demyelinating and (p)reactive lesions. Brain 124:1635-1645.

[37] Sachdev P, Chen X, Wen W (2008) White matter hyperintensities in mid-adult life. Curr Opin Psychiatry 21:268-274.

[38] Yue NC, Arnold AM, Longstreth WT Jr, Elster AD, Jungreis CA, O'Leary DH, Poirier VC, Bryan RN (1997) Sulcal, ventricular, and white matter changes at MR imaging in the aging brain: data from the cardiovascular health study. Radiology 202:33-39.

[39] Beggs CB. Venous hemodynamics in neurological disorders: an analytical review with hydrodynamic analysis (2013) BMC Med 11:142.

[40] Dolic K, Weinstock-Guttman B, Marr K, Valnarov V, Carl E, Hagemeier J, Kennedy C, Kilanowski C, Hojnacki D, Ramanathan M, Zivadinov R (2012) Heart disease, overweight, 
and cigarette smoking are associated with increased prevalence of extra-cranial venous abnormalities. Neurol Res 34:819-27.

[41] Fitzpatrick MA, Hinderliter AL, Egan BM, Julius S (1986) Decreased

venousdistensibility and reduced renin responsiveness in hypertension. Hypertension 8:II36-

43.

[42] Takeshita A, Mark AL (1979). Decreased venous distensibility in borderline hypertension. Hypertension 1:202-6.

[43] Klabunde RE (2004) Cardiovascular physiology concepts, eds. Lippincott Williams and Wilkins, Philadelphia, PA. 
Table 1 Clinical characteristics and MRI variables between control, MCI, and AD groups.

\begin{tabular}{lcccc}
\hline & $\begin{array}{c}\text { Control } \\
(\mathrm{n}=17)\end{array}$ & $\begin{array}{c}\text { MCI } \\
(\mathrm{n}=24)\end{array}$ & $\begin{array}{c}\text { AD } \\
(\mathrm{n}=12)\end{array}$ & $p$ \\
\hline Age, years, mean (SD) & $81.4(3.8)$ & $81.4(4.0)$ & $81.8(6.4)$ & 0.968 \\
Females, n (\%) & $5(29.4)$ & $11(45.8)$ & $6(50.0)$ & $0.457^{\mathrm{a}}$ \\
Education, years, mean(SD) & $13.4(1.8)$ & $10.9(4.4)$ & $9.8(4.1)$ & 0.033 \\
MMSE & $28.1(1.6)$ & $26.2(1.9)$ & $20.4(3.1)$ & $<0.001$ \\
Hypertension, n (\%) & $11(64.7)$ & $12(50.0)$ & $7(58.3)$ & $0.639^{\mathrm{a}}$ \\
Diabetes mellitus, n (\%) & $4(23.5)$ & $5(20.8)$ & $3(25.0)$ & $0.956^{\mathrm{a}}$ \\
Hyperlipidemia, n (\%) & $3(17.6)$ & $2(8.3)$ & $6(50.0)$ & $0.014^{\mathrm{a}}$ \\
Smoking, n (\%) & $1(5.9)$ & $1(4.2)$ & $0(0.0)$ & $0.709^{\mathrm{a}}$ \\
JVR & & & & \\
Positive ${ }^{\mathrm{c}}, \mathrm{n}(\%)$ & $11(64.7)$ & $15(62.5)$ & $7(58.3)$ & $0.941^{\mathrm{a}}$ \\
Severity score ${ }^{\mathrm{d}}$, mean (SD) & $1.8(0.7)$ & $1.9(0.8)$ & $1.8(0.8)$ & $0.864^{\mathrm{b}}$ \\
WMC number changes, mean (SD) & & & & \\
T2 WM lesions + DAWM & $28.1(16.6)$ & $44.9(30.6)$ & $42.6(18.6)$ & 0.091 \\
$\quad$ T2 WM lesions & $25.4(17.3)$ & $25.3(16.0)$ & $24.8(19.9)$ & 0.996 \\
$\quad$ DAWM & $18.2(21.2)$ & $33.4(38.5)$ & $31.3(11.6)$ & 0.246 \\
WMC volume changes, mean(SD) & & & & \\
T2 WM lesions + DAWM & $9.5(12.8)$ & $11.9(14.7)$ & $10.6(16.4)$ & 0.875 \\
$\quad$ T2 WM lesions & $8.6(12.8)$ & $10.6(14.7)$ & $9.5(16.3)$ & 0.909 \\
$\quad$ Subcortical & $2.5(5.6)$ & $2.2(3.4)$ & $2.2(4.2)$ & 0.975 \\
$\quad$ Periventricular & $6.1(7.8)$ & $8.4(13.8)$ & $7.4(12.1)$ & 0.828 \\
$\quad$ DAWM & $0.9(0.9)$ & $1.3(1.1)$ & $1.1(0.6)$ & 0.483 \\
$\quad$ Subcortical & $0.5(0.6)$ & $0.7(0.7)$ & $0.4(0.3)$ & 0.252 \\
$\quad$ Periventricular & $0.4(0.4)$ & $0.6(0.7)$ & $0.7(0.5)$ & 0.504 \\
Normalized LV volume & $77.4(27.0)$ & $79.5(24.8)$ & $91.8(25.3)$ & 0.294 \\
CSF volume & $374.5(54.4)$ & $364.0(63.8)$ & $367.0(63.4)$ & 0.860 \\
\hline$\quad$ & & &
\end{tabular}

$\mathrm{JVR}=$ jugular vein reflux $; \mathrm{n}=$ number $; \mathrm{SD}=$ standard deviation; $\%=$ percentage $; \mathrm{WM}=$ white matter; $\mathrm{WMC}=$ white matter changes; $\mathrm{MCI}=$ mild cognitive impairment; $\mathrm{AD}=$ Alzheimer's disease; DAWM = dirty-appearing white matter; $\mathrm{LV}=$ lateral ventricle; $\mathrm{CSF}=$ cerebrospinal fluid

$\mathrm{p}$ value determined by one-way ANOVA unless otherwise stated.

${ }^{a} p$ value determined using chi square test.

${ }^{b} p$ value determined using Kruskal-Wallis test.

${ }^{\mathrm{c}}$ The presence of JVR in either side would be defined as JVR positive dJVR severity score: $1=$ no JVR; $2=$ mild JVR; $3=$ severe JVR

Table 2. Clinical characteristics and MRI variables between three JVR-graded groups. 


\begin{tabular}{lcccc}
\hline & $\begin{array}{c}\text { No JVR } \\
(\mathrm{n}=20)\end{array}$ & $\begin{array}{c}\text { Mild JVR } \\
(\mathrm{n}=28)\end{array}$ & $\begin{array}{c}\text { Severe JVR } \\
(\mathrm{n}=5)\end{array}$ & $p$ \\
\hline Age, years, mean (SD) & $81.1(3.4)$ & $81.7(5.0)$ & $81.6(6.3)$ & 0.882 \\
Females, n (\%) & $8(40.0)$ & $13(46.4)$ & $1(20.0)$ & $0.535^{\mathrm{a}}$ \\
Disease classification, n (\%) & & & $0.864^{\mathrm{b}}$ \\
$\quad$ Control & $6(30.0)$ & $10(35.7)$ & $1(20.0)$ & \\
MCI & $9(45.0)$ & $12(42.9)$ & $3(60.0)$ & \\
AD & $5(25.0)$ & $6(21.4)$ & $1(20.0)$ & \\
Education, years, mean (SD) & $12.3(4.0)$ & $10.9(3.8)$ & $11.2(4.2)$ & 0.496 \\
MMSE & $25.1(4.0)$ & $25.9(3.1)$ & $24.8(4.7)$ & 0.705 \\
Hypertension, n (\%) & $8(40.0)$ & $17(60.7)$ & $5(100.0)$ & $0.044^{\mathrm{a}}$ \\
Diabetes mellitus, n (\%) & $7(35.0)$ & $5(17.9)$ & $0(0.0)$ & $0.168^{\mathrm{a}}$ \\
Hyperlipidemia, n (\%) & $4(20.0)$ & $6(21.4)$ & $1(20.0)$ & $0.992^{\mathrm{a}}$ \\
Smoking, (\%) & $0(0.0)$ & $2(7.1)$ & $0(0.0)$ & $0.395^{\mathrm{a}}$ \\
WMC number changes, mean (SD) & & & & \\
T2 WM lesions + DAWM & $35.4(20.8)$ & $38.1(26.9)$ & $58.0(26.6)$ & 0.193 \\
$\quad$ T2 WM lesions & $23.1(15.9)$ & $25.7(19.1)$ & $31.0(3.9)$ & 0.642 \\
DAWM & $28.6(27.4)$ & $24.3(32.2)$ & $46.6(13.6)$ & 0.301 \\
WMC volume changes, mean (SD) & & & & \\
T2 WM lesions + DAWM & $7.3(8.9)$ & $11.3(15.2)$ & $21.9(22.2)$ & 0.119 \\
$\quad$ T2 WM lesions & $6.2(8.9)$ & $10.4(15.2)$ & $19.8(22.6)$ & 0.150 \\
$\quad$ Subcortical & $1.5(3.2)$ & $2.6(4.7)$ & $4.1(6.0)$ & 0.441 \\
$\quad$ Periventricular & $4.7(7.4)$ & $7.9(12.6)$ & $15.7(17.0)$ & 0.159 \\
DAWM & $1.1(0.8)$ & $0.9(1.0)$ & $2.1(1.0)$ & 0.047 \\
$\quad$ Subcortical & $0.5(0.4)$ & $0.6(0.7)$ & $1.0(0.3)$ & 0.296 \\
$\quad$ Periventricular & $0.7(0.5)$ & $0.4(0.4)$ & $1.1(1.0)$ & 0.008 \\
Normalized LV volume & $81.6(25.9)$ & $77.8(24.4)$ & $102.8(27.4)$ & 0.136 \\
CSF volume & $373.0(53.0)$ & $355.1(61.6)$ & $420.7(53.3)$ & 0.067 \\
\hline$\quad$ & & &
\end{tabular}

$\mathrm{JVR}=$ jugular vein reflux; $\mathrm{n}=$ number; $\mathrm{SD}=$ standard deviation; $\%$ - percentage; $\mathrm{WM}=$ white matter; $\mathrm{WMC}=$ white matter changes; $\mathrm{MCI}=$ mild cognitive impairment; $\mathrm{AD}=$ Alzheimer's disease; DAWM = dirty-appearing white matter; LV = lateral ventricle; CSF = cerebrospinal fluid

Volumes are expressed in millilitres.

$\mathrm{p}$ value determined by one-way ANOVA unless otherwise stated.

${ }^{a} p$ value determined using chi square test.

${ }^{\mathrm{b}} \mathrm{p}$ value determined using Kruskal-Wallis test.

Table 3. MRI variables classified according to JVR status (i.e. positive or negative). 


\begin{tabular}{|c|c|c|c|}
\hline & JVR negative & JVR positive & $p$ \\
\hline Controls, n (\%) & $6(30.0)$ & $11(70.0)$ & NA \\
\hline \multicolumn{4}{|c|}{ WMC number changes, mean (SD) } \\
\hline T2 WM lesions + DAWM & $24.2(16.7)$ & $30.3(17.0)$ & 0.509 \\
\hline T2 WM lesions & $21.5(14.3)$ & $27.5(19.1)$ & 0.713 \\
\hline DAWM & $13.8(10.3)$ & $20.5(25.4)$ & 0.831 \\
\hline \multicolumn{4}{|c|}{ WMC volume changes, mean (SD) } \\
\hline T2 WM lesions + DAWM & $3.9(3.4)$ & $12.6(15.1)$ & 0.216 \\
\hline T2 WM lesions & $3.3(3.1)$ & $11.5(15.2)$ & 0.256 \\
\hline Subcortical & $0.7(0.9)$ & $3.5(6.9)$ & 0.538 \\
\hline Periventricular & $2.6(2.5)$ & $8.0(9.1)$ & 0.216 \\
\hline DAWM & $0.6(0.4)$ & $1.1(1.1)$ & 0.612 \\
\hline Subcortical & $0.3(0.2)$ & $0.6(0.7)$ & 0.675 \\
\hline Periventricular & $0.4(0.3)$ & $0.4(0.4)$ & 0.904 \\
\hline Normalized LV volume & $69.0(23.1)$ & $82.0(28.9)$ & 0.525 \\
\hline CSF volume & $348.0(49.7)$ & $389.0(53.4)$ & 0.216 \\
\hline MCI, n (\%) & $9(45.0)$ & $15(55.0)$ & NA \\
\hline \multicolumn{4}{|c|}{ WMC number changes, mean (SD) } \\
\hline T2 WM lesions + DAWM & $35.4(19.9)$ & $50.5(35.0)$ & 0.633 \\
\hline T2 WM lesions & $26.3(18.2)$ & $24.7(15.1)$ & 0.743 \\
\hline DAWM & $37.9(37.6)$ & $30.7(40.0)$ & 0.455 \\
\hline \multicolumn{4}{|c|}{ WMC volume changes, mean (SD) } \\
\hline T2 WM lesions + DAWM & $11.4(12.0)$ & $12.2(16.4)$ & 0.952 \\
\hline T2 WM lesions & $10.1(12.2)$ & $10.9(16.4)$ & 1.000 \\
\hline Subcortical & $2.7(4.6)$ & $2.0(2.6)$ & 0.905 \\
\hline Periventricular & $7.4(10.4)$ & $9.0(15.7)$ & 0.858 \\
\hline DAWM & $1.3(1.0)$ & $1.3(1.2)$ & 0.698 \\
\hline Subcortical & $0.7(0.6)$ & $0.7(0.8)$ & 0.952 \\
\hline Periventricular & $0.6(0.6)$ & $0.5(0.8)$ & 0.426 \\
\hline Normalized LV volume & $85.8(29.6)$ & $75.6(21.6)$ & 0.474 \\
\hline CSF volume & $377.3(54.2)$ & $356.0(69.4)$ & 0.512 \\
\hline $\mathrm{AD}, \mathrm{n}(\%)$ & $5(25.0)$ & $7(75.0)$ & NA \\
\hline \multicolumn{4}{|c|}{ WMC number changes, mean (SD) } \\
\hline T2 WM lesions + DAWM & $48.8(22.5)$ & $38.1(15.5)$ & 0.505 \\
\hline T2 WM lesions & $19.2(15.5)$ & $28.9(22.7)$ & 0.530 \\
\hline DAWM & $29.4(8.6)$ & $32.6(13.9)$ & 0.861 \\
\hline \multicolumn{4}{|c|}{ WMC volume changes, mean (SD) } \\
\hline T2 WM lesions + DAWM & $4.2(2.2)$ & $15.2(20.7)$ & 0.202 \\
\hline T2 WM lesions & $2.8(1.8)$ & $14.4(20.4)$ & 0.073 \\
\hline Subcortical & $0.3(0.4)$ & $3.5(5.2)$ & 0.073 \\
\hline Periventricular & $2.5(1.6)$ & $10.9(15.2)$ & 0.073 \\
\hline DAWM & $1.4(0.7)$ & $0.8(0.5)$ & 0.106 \\
\hline Subcortical & $0.3(0.3)$ & $0.4(0.4)$ & 0.755 \\
\hline Periventricular & $1.1(0.4)$ & $0.4(0.2)$ & 0.010 \\
\hline Normalized LV volume & $88.9(20.6)$ & $93.8(29.7)$ & 0.876 \\
\hline CSF volume & $395.4(53.0)$ & $346.8(66.2)$ & 0.268 \\
\hline
\end{tabular}


JVR $=$ jugular vein reflux $; \mathrm{n}=$ number; $\mathrm{SD}=$ standard deviation; $\%$ - percentage $; \mathrm{WM}=$ white matter; $\mathrm{WMC}=$ white matter changes; $\mathrm{MCI}=$ mild cognitive impairment; $\mathrm{AD}=$ Alzheimer's disease; DAWM = dirty-appearing white matter; LV = lateral ventricle; $\mathrm{CSF}=$ cerebrospinal fluid; NA - non available.

Volumes are expressed in millilitres.

p values determined by 2-tailed Mann Whitney U-test. 


\section{FIGURE LEGENDS}

Fig. 1. Examples of periventricular and subcortical white matter T2 lesions and dirty appearing white matter in controls, mild cognitive impairment patients and Alzheimer's disease patients.

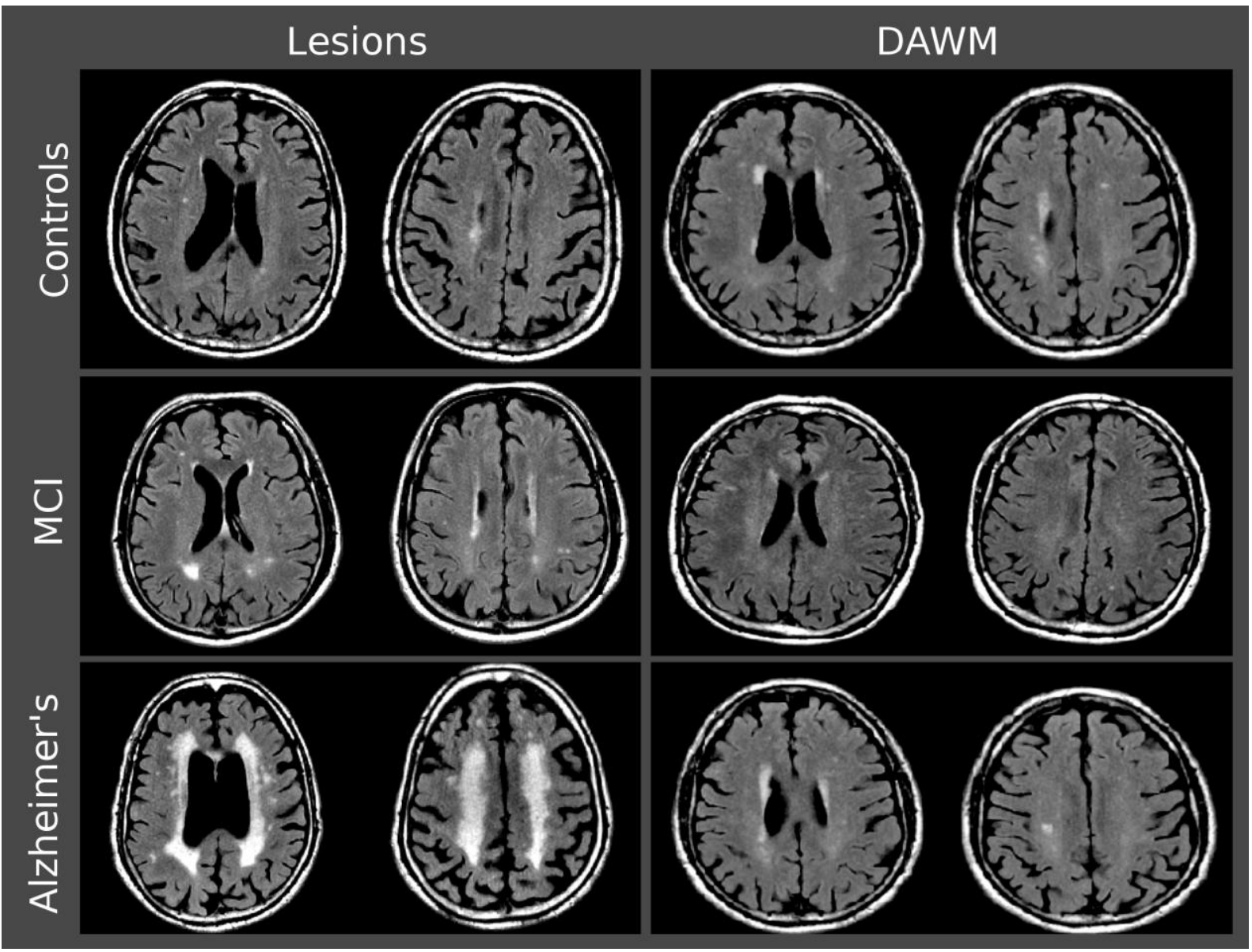


Fig 2. Plot of the logit vs. logistic function for the AD patients group using the variables periventricular T2 WMC volume $(\mathrm{B}=0.0004)$ and periventricular DAWM volume $(\mathrm{B}=-$ 0.0039). Logit $=0.5$ is deemed to separate the JVR positive subjects from the negative subjects. This results in one false positive and one false negative (sensitivity $=85.7 \%$ and specificity $=80 \%)$.

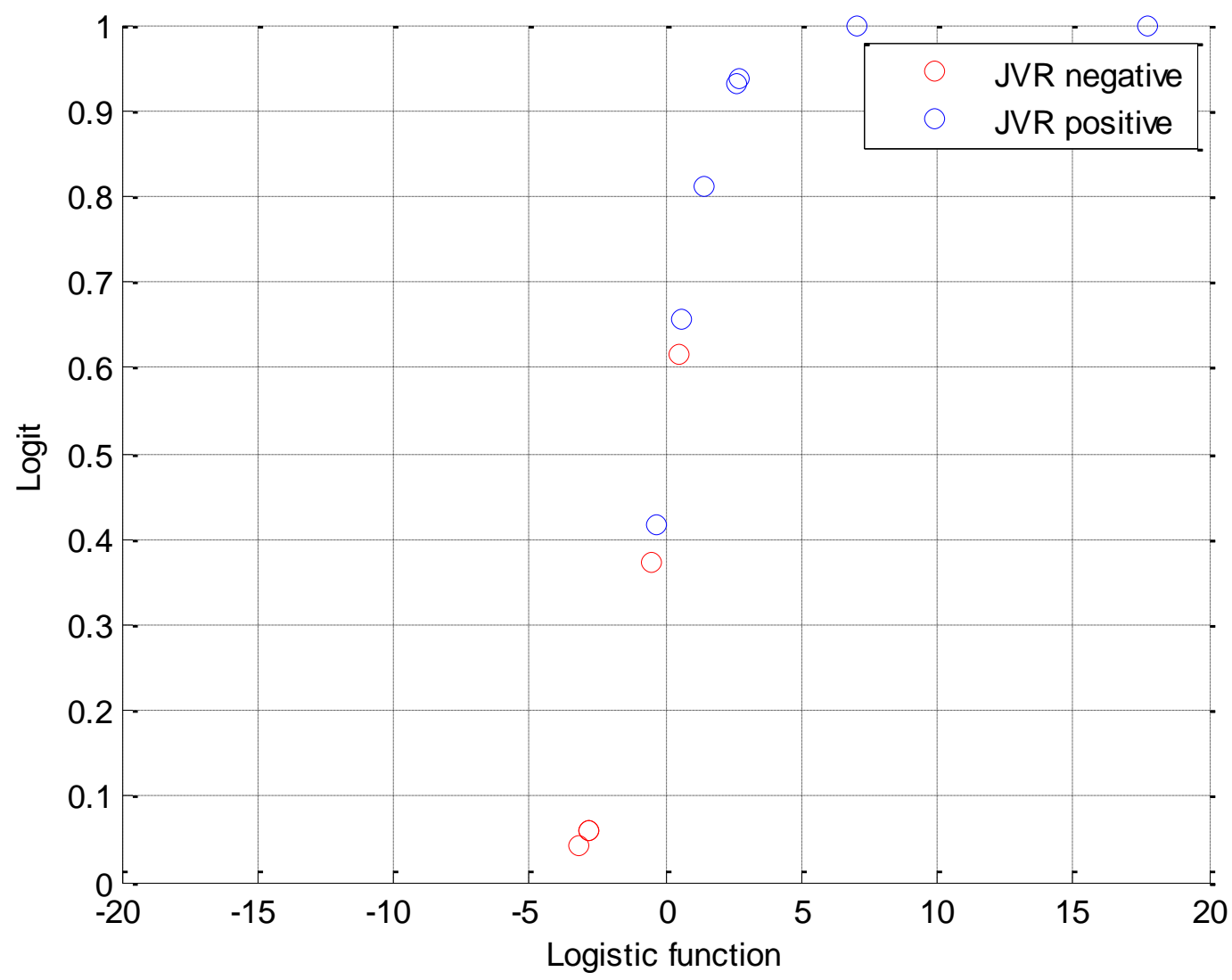

$\mathrm{JVR}=$ jugular vein reflux; $\mathrm{AD}=$ Alzheimer's disease; $\mathrm{WMC}=$ white matter change; DAWM $=$ dirty-appearing white matter 


\section{SUPPLEMENTARY DATA}

\section{Supplementary Appendix 1: The definitions of vascular risk factors}

Hypertension was defined as a history of hypertension, use of antihypertensive medications, or a measured blood pressure consistently $>140 / 90 \mathrm{~mm} \mathrm{Hg}$. Hyperlipidemia was defined as a cholesterol level >200 mg/dL, low density lipoprotein >150 mg/dL, triglyceride level >150 $\mathrm{mg} / \mathrm{dL}$, or history of hyperlipidemia. Diabetes was defined as a history of diabetes, use of medications for diabetes, or an elevated fasting blood glucose $>126 \mathrm{mg} / \mathrm{dL}$.

\section{Supplementary Appendix 2: Color-coded duplex ultrasonography: JVR determination}

Neck color-coded duplex sonography was performed in all subjects with a 7-MHz linear transducer (iU22; Philips, New York, NY, USA) by the same technician, who was blinded to subjects' characteristics. On examination, subjects were in a head-straight, flat supine position after a quiet $10 \mathrm{~min}$ rest. The IJV was initially insonated longitudinally and thoroughly from the proximal part of the neck base rostrally to the distal part at the submandibular level in order to detect any possible spontaneous JVR at baseline. Then, the VM was performed by forcible expiration from subject's mouth into a flexible rubber tube connected to a manometer. Subjects were asked to reach $40 \mathrm{~mm} \mathrm{Hg}$ Valsalva pressure and maintain it for at least 10 seconds. During the VM, the distal margin of the window of the color signal was placed at the tip of the flow divider of the internal carotid artery. The color box was adjusted to include the entire lumen of the IJV; if retrograde color appeared in the center of the lumen, the retrograde flow would then be confirmed by Doppler spectrum. JVR was determined when the retrograde-flow color in the center of the lumen and the Doppler-flow waveform demonstrated reversal of flow for more than 0.5 seconds. 